\title{
Uniform quantized electron gas: Radiation corrections
}

\author{
Johan S. Høye ${ }^{1}$ and Enrique Lomba ${ }^{2}$ \\ ${ }^{1}$ Department of Physics, Norwegian University of \\ Science and Technology, N-7491 Trondheim, Norway \\ 2 Instituto de Qímica Física Rocasolano, \\ CSIC, Serrano 119, E-28006 Madrid, Spain
}

(Dated: April 30, 2022)

\begin{abstract}
In this paper we analyze how radiation effects influence the correlation functions, the excess energy, and in turn the electron correlation energy of the quantized electron gas at temperature $T=0$. To that aim we resort to a statistical mechanical description of the quantum problem of electron correlations, based on the path integral formalism. In previous works we studied and found accurate results for the usual situation with the electrostatic Coulomb interaction. Here the additional problem with radiation is taken into account. This is facilitated by the equivalence to a dielectric fluid for which correlation functions for dipolar moments are established. From these functions follows the usual density-density (or charge-charge) correlation function needed for the longitudinal electrostatic problem, and in addition the one needed for the transverse radiation problem. While electrostatic excess energy is negative, the transverse one is positive. This quantity is small and decreases rapidly for decreasing densities. However, for high densities it approaches the electrostatic contribution, eventually becoming even larger. The part of the transverse energy from induced correlations turns out to be very small. Also, the non-local longitudinal and transverse dielectric constants of the electron gas are identified from the induced correlation functions.
\end{abstract}




\section{INTRODUCTION}

Electron correlation energy is a fundamental quantity in quantum chemistry calculations. Standard procedures for its evaluation are Configuration Interaction approaches or manybody perturbation theories [1]. In a series of works [2 4] the authors have developed an alternative approach based on the standard procedures of classical statistical mechanics using the path integral formalism, which translates the quantum problem into a classical polymer problem in four dimensions. The well known RPA (random phase approximation) was recovered as a basic result [3]. Then in Ref. [4] a much more accurate approximation was derived starting from the enforcement of thermodynamic consistency.

In this paper we analyze and evaluate how radiation effects influence the excess and correlation energies of the uniform quantized electron gas. These results can also provide an indication of the significance of these effects for electrons in molecules.

In this connection, quantum computations require the use of non-zero frequencies of the interaction. This implies that the Coulomb interaction has to be extended in a non-trivial way, since it must involve the electromagnetic vector potential. This again means that current-current correlations have to be taken into account. This situation was analyzed in Ref. [2] where it was found that the problem can be simplified by introducing polarizations, through which charge densities and currents can be expressed. In this way the problem is transformed into that of a dielectric fluid with radiating dipole-dipole interaction.

As shown in Sec. III the dipole interaction can be separated into two components that do not couple. This simplifies considerably in our case since one part will recover precisely the standard electrostatic result, by which the remaining contribution is entirely due to radiation. This separation of the dipolar interaction has been utilized earlier in theories for classical dielectric fluids. It was already used by Wertheim to solve the MSA (Mean Spherical Approximation) for dipolar hard spheres where both terms are present in the electrostatic

case [5, 6] as well. The same two terms also occur in the study of the refractive index of polarizable fluids with radiating interaction [7]. Note that this uncoupling is just a particular case of the general rotational invariant expansion proposed by Blum and Torruella [8] to deal with two-body correlations in molecular fluids.

As already mentioned, one part of the interaction and the resulting correlation function of the electron gas is tied to the longitudinal electrostatic interaction. The other part is 
connected to the transverse electromagnetic radiation. The first part, the usual electrostatic problem, was studied in Refs. [3, 4] where it was shown that the approach leads accurate results. In the present case the additional contribution from the transverse frequency dependent interaction is included. This is obtained by extension of the statistical mechanical method introduced in Refs. [3, 4]. This method also coincides with the well-known RPA when effective interaction is not used. The additional problem with the transverse contribution is the need for current correlations for the free electron gas. However, expressions for these follow from Ref. [2].

By our computations we find that radiations effects are relatively small compared to the the contribution due to the usual electrostatic interaction alone. This is consistent with the previous study of polarizable hard spheres where the positions of the spheres were treated classically while their oscillating dipole moments were quantized [9]. This is also consistent with previous results for induced Casimir energies that are known to be responsible for the attraction between dielectric media. These energies are usually attributed to quantum fluctuations of the electromagnetic field [10]. But this field can be eliminated to be replaced with radiating dipole interaction between polarizable particles [11. For a pair of polarizable particles one, for the Casimir energy, finds an induced $1 / r^{6}$ van der Waals interaction for small separations while for large separations the faster $1 / r^{7}$ decay takes place due to retardation (radiation) effects [10, 11]. Thus, radiation reduces the induced interaction for large $r$, but when integrating over it the total net effect of radiation is small. Anyway, our analysis of the transverse exchange energy shows that the influence of radiation effects is most visible for high electron densities.

The rest of the paper is organized as follows. In Section II we briefly review the expressions that define the correlation functions for the electron gas in the path integral formalism. The radiating dipole interaction potential is presented in Section III. In Sections IV and V we find the exchange and correlation electron energies. First we recover previous expressions for the longitudinal electrostatic case. Then these expressions are extended to the new situation where radiation is included by which the additional transverse contribution is obtained. The dielectric constant of the electron gas is discussed in Section VI. Finally in Section VII numerical results are presented. The expressions for analytic integration of the transverse exchange energy are explicitly detailed in the Appendix. 


\section{CORRELATION FUNCTIONS}

The charge-charge (or density-density) correlation function of the free fermion gas and the electrostatic Coulomb potential were needed to obtain the correlation energy of the uniform electron gas. However, there will be radiation corrections that are expected to be small. These we will incorporate in a quantitative way by extension of the method used to obtain our previous results for the correlation energy. This extension will require currentcurrent correlations to take into account the electromagnetic vector potential that appears when time-dependent electromagnetic forces are taken into account. This has been studied on a more general basis in Ref. [2]. There it was noticed that the electron problem can be regarded and transformed to the problem of a dielectric fluid.

The transformation to a dielectric fluid simplifies the problem since both charge density $\rho$ and current density $\mathbf{j}$ can be expressed in terms of polarization $\mathbf{P}$. One has

$$
\rho=-\nabla \mathbf{P} \quad \text { and } \quad \mathbf{j}=\frac{\partial \mathbf{P}}{\partial t}
$$

This is Eq. (I18) of Ref. [2]. For simplicity equations of this reference are preceded by the numeral I when referred to. With this Eq. (I14), the equation of charge conservation $\partial \rho / \partial t+\nabla \mathbf{j}=0$ is fulfilled. By Fourier transform (signs depending upon convention used)

$$
\nabla \rightarrow-i \mathbf{k}, \quad \frac{\partial}{\partial t} \rightarrow i \omega
$$

The Fourier transform of the correlation function in imaginary time and space for a pair of polarizations $\mathbf{s}_{1}$ and $\mathbf{s}_{2}$ of the uniform free electron gas is given by Eq. (I67) (for unit charges, $i, j=x, y, z)$

$$
\begin{aligned}
\left\langle s_{1 i} s_{2 j}\right\rangle=\hat{P}_{i j}(K, \mathbf{k}) & =\int\left[\frac{\hbar}{2 m}\left(k_{i}^{\prime \prime}-k_{i}^{\prime}\right)\right]\left[\frac{\hbar}{2 m}\left(k_{j}^{\prime \prime}-k_{j}^{\prime}\right)\right] \frac{\hbar^{2}}{\Delta^{2}+K^{2}} L d \mathbf{k}^{\prime}, \\
L & =\frac{\zeta}{(2 \pi)^{3}} \frac{X-Y}{\Delta(1 \pm \zeta X)(1 \pm \zeta Y)} .
\end{aligned}
$$

The Matsubara frequency $K=i \hbar \omega$ ( $\omega$ is frequency) is the Fourier variable in imaginary time. Further $\zeta=\exp (\beta \mu)$ where $\mu$ is chemical potential, $\beta=1 /\left(k_{B} T\right)$, and $k_{B}$ is Boltzmann's constant. In the expression for $L$ the $+\operatorname{sign}$ is for fermions. Further

$$
\begin{gathered}
X=F_{\beta}\left(k^{\prime}\right), \quad Y=F_{\beta}\left(k^{\prime \prime}\right), \quad \text { with } \quad F_{\lambda}(k)=\exp (-\lambda E(k)), \\
E(k)=\frac{(\hbar k)^{2}}{2 m}, \quad \Delta=E\left(k^{\prime \prime}\right)-E\left(k^{\prime}\right), \quad \mathbf{k}^{\prime \prime}=\mathbf{k}-\mathbf{k}^{\prime}
\end{gathered}
$$


The imaginary time $\lambda$ is limited to $0 \leq \lambda \leq \beta$. Details of the derivation of expression (3) can be found in Ref. [2]. However, one notes that the known density-density correlation function (I69) follows as (where $\sum_{i j}$ is implicit)

$$
\hat{S}(K, k)=k_{i} k_{j} \hat{P}_{i j}(K, k)=\int \frac{\Delta^{2}}{\Delta^{2}+K^{2}} L d \mathbf{k}^{\prime} .
$$

This is also Eq. (5) in Ref. [4] -Eq. (II5)-. Hereafter the numeral II will be used to designate the equations of Ref. [4]. Note that due to symmetry one can perform the substitution $1 /(i K+\Delta) \rightarrow[1 /(\Delta+i K)+1 /(\Delta-i K)] / 2=\Delta /\left(\Delta^{2}+K^{2}\right)$.

\section{RADIATING DIPOLE INTERACTION}

The radiating dipole-dipole pair interaction between two dipole moments $\mathbf{s}_{1}$ and $\mathbf{s}_{2}$ (per unit charge) is given by Eq. (I24)

$$
\begin{aligned}
\hat{\phi}(12, \omega) & =\hat{\psi}(k, \omega)\left[\left(\mathbf{k} \cdot \mathbf{s}_{1}\right)\left(\mathbf{k} \cdot \mathbf{s}_{2}\right)-\left(\frac{\omega}{c}\right)^{2} \mathbf{s}_{1} \cdot \mathbf{s}_{2}\right], \\
\hat{\psi}(k, \omega) & =\frac{e^{2}}{\varepsilon_{0}\left(k^{2}-(\omega / c)^{2}\right)} .
\end{aligned}
$$

Here SI units are used instead of Gaussian ones $\left(4 \pi \rightarrow 1 / \varepsilon_{0}\right)$. The $-e$ is the electric charge of the electron, $\varepsilon_{0}$ is the permittivity of vacuum, and $\omega$ is the frequency.

Now one notes that interaction (8) can be written in the form

$$
\begin{gathered}
\hat{\phi}(12, \omega)=\tilde{\psi}(k)\left[J_{1}-\frac{(\omega / c)^{2}}{k^{2}-(\omega / c)^{2}} J_{2}\right], \quad \text { with } \quad \tilde{\psi}(k)=\frac{e^{2}}{\varepsilon_{0} k^{2}}, \\
J_{1}=\left(\mathbf{k} \cdot \mathbf{s}_{1}\right)\left(\mathbf{k} \cdot \mathbf{s}_{2}\right), \quad J_{2}=k^{2} \mathbf{s}_{1} \cdot \mathbf{s}_{2}-\left(\mathbf{k} \cdot \mathbf{s}_{1}\right)\left(\mathbf{k} \cdot \mathbf{s}_{2}\right)=\left(\mathbf{k} \times \mathbf{s}_{1}\right)\left(\mathbf{k} \times \mathbf{s}_{2}\right) .
\end{gathered}
$$

With $\tilde{\psi}(k)$ alone we are back to the electrostatic Coulomb interaction. To obtain the resulting correlation function and correlation energy one has to perform convolutions as in the electrostatic case of Ref. 4]. As will be seen the correlation function (3) can be separated in $J_{1}$ and $J_{2}$ terms. A convolution (an orientational average) needed leads to

$$
\begin{gathered}
J_{1} J_{1}=\left(\mathbf{k} \cdot \mathbf{s}_{1}\right)\left\langle\left(\mathbf{k} \cdot \mathbf{s}_{3}\right)\left(\mathbf{k} \cdot \mathbf{s}_{4}\right)\right\rangle\left(\mathbf{k} \cdot \mathbf{s}_{2}\right)=S_{L} J_{1} \\
\left\langle J_{1}\right\rangle=S_{L}, \quad S_{L}=\hat{S}_{L}(K, k)=\hat{S}(K, k)
\end{gathered}
$$

where $\tilde{S}(K, k)$ is the correlation function $(7)$. Altogether with the $J_{1}$ term alone the RPA of the electrostatic case is recovered. Also the $\tilde{\psi}(k)$ can be replaced by an effective (or cut) interaction as determined in Ref. [4] to obtain accurate results. 
To include radiation the convolutions $J_{1} J_{2}$ and $J_{2} J_{2}$ are needed. One notes that from expression (3) the matrix formed by $\hat{P}_{i j}=\hat{P}_{i j}(K, k)$ can be made diagonal. With the $z$ axis along the vector $\mathbf{k}=\{0,0, k\}$ one has $\tilde{P}_{i j}=0$ with $i \neq j$ and $\tilde{P}_{x x}=\tilde{P}_{y y}\left(\right.$ since $\left.k_{x}=k_{y}=0\right)$. With this

$$
J_{1}=k^{2} s_{1 z} s_{2 z} \quad \text { and } \quad J_{2}=k^{2}\left(s_{1 x} s_{2 x}+s_{1 y} s_{2 y}\right) .
$$

Accordingly $J_{1} J_{2}=0$ and

$$
\begin{gathered}
J_{2} J_{2}=k^{4}\left[s_{1 x}\left\langle s_{3 x} s_{4 x}\right\rangle s_{2 x}+s_{1 y}\left\langle s_{3 y} s_{4 y}\right\rangle s_{2 y}\right]=S_{T} J_{2}, \\
\left\langle J_{2}\right\rangle=k^{2}\left[\left\langle s_{1 x} s_{2 x}\right\rangle+\left\langle s_{1 y} s_{2 y}\right\rangle\right]=2 S_{T}, \quad S_{T}=\hat{S}_{T}(K, k)=k^{2} \hat{P}_{x x}=k^{2} \hat{P}_{y y} .
\end{gathered}
$$

Since $J_{1} J_{2}=0$ the contribution from radiation decouples from the electrostatic result. By that the computations can be performed in a way similar to the electrostatic part, but with an interaction that follows from Eq. (9) and a reference system correlation function given by $\hat{S}_{T}(K, k)$ which couples to the transverse radiating field. Likewise $S_{L}=\hat{S}_{L}(K, k)$ is connected to the longitudinal electrostatic field as before.

\section{EXCHANGE ENERGIES}

In this section we will provide expressions to evaluate the exchange energy contribution to the electron energy, due to both electrostatic and radiation contributions.

\section{A. Electrostatic exchange energy}

The exchange energy follows by integrating the interaction together with the correlation function and divide by two to avoid double counting. For the electrostatic case the equal (imaginary) time correlation function $\tilde{S}_{L}(0, k)=\tilde{S}(0, k)$ is needed. Also the self correlation given by particle density $\rho$ should be subtracted to avoid divergence. The electrostatic exchange energy per unit volume is thus

$$
F_{\text {Lex }}=\frac{1}{2(2 \pi)^{3}} \int(g \tilde{S}(0, k)-\rho) \tilde{\psi}(k) d \mathbf{k}
$$

where $g=2$ is the spin degeneracy. With $g \tilde{S}(0, k)=\rho\left(3 Q / 2-Q^{3} / 2\right)$ for $Q<1$ and equal to $\rho$ for larger $Q$ where $Q=k /\left(2 k_{f}\right)[3]$ one finds the known answer Eq. (II22)

$$
F_{L e x}=-\frac{3 \pi e^{2} k_{f}}{2(2 \pi)^{3} \varepsilon_{0}} \rho=12 \rho \mu_{f} D I_{L e x}, \quad \text { with } \quad I_{\text {Lex }}=-\frac{1}{4},
$$


and where

$$
\begin{aligned}
\rho & =\frac{4 \pi g}{3(2 \pi)^{3}} k_{f}^{3}, \quad \frac{4 \pi}{3}\left(r_{s} a_{0}\right)^{3}=\frac{1}{\rho}, \quad a_{0}=\frac{4 \pi \varepsilon_{0} \hbar^{2}}{m e^{2}}, \\
\mu_{f} & =\frac{\left(\hbar k_{f}\right)^{2}}{2 m}=\frac{50.1 \mathrm{eV}}{r_{s}^{2}}, \quad \text { and } \quad D=\frac{m k_{f}}{2 \pi^{2} \hbar^{2}} \frac{e^{2}}{\varepsilon_{0}\left(2 k_{f}\right)^{2}}=0.082393 \cdot r_{s}
\end{aligned}
$$

is used [3, 4]. Here $r_{s}$ is the dimensionless length parameter commonly used, $a_{0}$ is the Bohr radius, $k_{f}$ the Fermi wave vector, and $\mu_{f}$ is the Fermi energy which also is the chemical potential of the free electron gas at $T=0$.

Now the exchange energy also may be found by rearranging integrations. This will be useful for its radiation exchange contribution by which analytical evaluation of it becomes possible too. At $T=0$ the Matsubara frequencies $K=2 \pi n / \beta$ (with $n$ integer) become continuous, and we have $\left(2 \pi \sum_{n} \rightarrow \beta d K\right)$

$$
\tilde{S}(0, k)=\frac{1}{2 \pi} \int_{-\infty}^{\infty} \hat{S}(K, k) d K
$$

where $\hat{S}(K, k)$ is given by integral $(7)$. First we write it as

$$
\hat{S}(K, k)=\frac{2 \pi}{(2 \pi)^{3}} \frac{2 m}{\hbar^{2}} \cdot 2 \int_{0<k^{\prime}<k_{f}} \int_{-1}^{1} \frac{\left(k^{2}-2 \mathbf{k k}^{\prime}\right)}{G^{2}+\left(k^{2}-2 \mathbf{k k}^{\prime}\right)^{2}} d(-\cos \theta) k^{\prime 2} d k^{\prime} .
$$

Here the factor 2 is from the exchange of roles of $X$ and $Y$ in expression (4) for $L$. Further $G=2 m K / \hbar^{2}$ and $\mathbf{k k}^{\prime}=k k^{\prime} \cos \theta$. The boundary $k_{f}$ for $k^{\prime}$ is obvious for $k>2 k_{f}$. For $k<2 k_{f}$ it can still be used due to contributions that from symmetry must cancel where both $k^{\prime \prime}, k^{\prime}<k_{f}$. Now quantities

$$
z=-\cos \theta, \quad t=\frac{k^{\prime}}{k_{f}}, \quad Q=\frac{k}{2 k_{f}}, \quad x=\frac{G}{2 k k_{f}}=\frac{m K}{\hbar^{2} k k_{f}}=\frac{K}{4 \mu_{f} Q}
$$

are introduced to obtain

$$
\begin{gathered}
\hat{S}_{L}(K, k)=\hat{S}(K, k)=S_{L}(Q, x)=\frac{m k_{f}}{4 \pi^{2} \hbar^{2}} I_{L}(Q, x), \\
I_{L}(Q, x)=\frac{1}{Q} \int_{0}^{1}\left[\int_{-1}^{1} \frac{Q+t z}{x^{2}+(Q+t z)^{2}} d z\right] t^{2} d t .
\end{gathered}
$$

This together with the Coulomb interaction $(10)\left(\sim 1 / Q^{2}\right)$ inserted in Eqs. 16) and 17 together with 19 gives $\left(\int_{0}^{1} \int_{-1}^{1} d z t^{2} d t=2 / 3\right)$

$$
I_{\text {Lex }}=\frac{1}{\pi} \int_{0}^{\infty}\left[\int_{-\infty}^{\infty} I_{L}(Q, x) Q d x-\frac{2}{3}\right] \frac{1}{Q^{2}} Q^{2} d Q=\int_{0}^{1} \int_{-1}^{1}\left[\int_{0}^{\infty} I_{L}(Q) d Q\right] t^{2} d z d t
$$




$$
I_{L}(Q)=\frac{a}{|a|}-1, \quad a=Q+t z .
$$

Nonzero $I_{L}(Q)$ requires $Q+t z<0$, i.e. $Q<-t z$. Thus altogether (with $-t z>0$ )

$$
\begin{gathered}
J_{L}=\int_{0}^{\infty} I_{L}(Q) d Q=\int_{0}^{-t z}(-2) d Q=2 t z, \\
I_{L e x}=\int_{0}^{1} \int_{-1}^{0} J_{L} t^{2} d z d t=-\frac{1}{4}
\end{gathered}
$$

consistent with result (17).

\section{B. Radiation exchange energy}

The various elements of the correlation function matrix $P_{i j}$, given by (3), differ by the factor

$$
M_{i j}=\left(k_{i}^{\prime \prime}-k_{i}^{\prime}\right)\left(k_{j}^{\prime \prime}-k_{j}^{\prime}\right)=\left(k_{i}-2 k_{i}^{\prime}\right)\left(k_{j}-2 k_{j}^{\prime}\right)
$$

in their integrands. Here $\mathbf{k}^{\prime \prime}=\mathbf{k}-\mathbf{k}^{\prime}$ is used. With the $z$ axis directed along $\mathbf{k}=\{0,0, k\}$ there will be no contributions to integral $(3)$ for $i \neq j$. Further $\left(\sum_{i j}\right)$

$$
\begin{gathered}
k^{2} M_{z z}=k_{i} k_{j} M_{i j}=\left(k^{2}-2 \mathbf{k k}^{\prime}\right)^{2}=k^{4}-4 k^{2} \mathbf{k k}^{\prime}+4\left(\mathbf{k k}^{\prime}\right)^{2} \\
k^{2}\left(M_{x x}+M_{y y}+M_{z z}\right)=k^{2}\left[\left(-2 k_{x}^{\prime}\right)^{2}+\left(-2 k_{y}^{\prime}\right)^{2}+\left(k-2 k_{z}^{\prime}\right)^{2}\right]=k^{2}\left(k^{2}-4 \mathbf{k} \mathbf{k}^{\prime}+4 k^{\prime 2}\right) \\
k^{2} M_{x x}=k^{2} M_{y y}=2\left(k^{2} k^{\prime 2}-\left(\mathbf{k k}^{\prime}\right)^{2}\right)
\end{gathered}
$$

Accordingly to compute the transverse correlation function $S_{T}=\hat{S}_{T}(K, k)$ the factor $k^{2} M_{z z}$ in the integral for the longitudinal one $S_{L}=\hat{S}_{L}(K, k)$ is replaced by $k^{2} M_{x x}$. It occurs twice as it has a $y$ component too.

Thus with new quantities (21) inserted in 29) and (31) we find the ratio

$$
\frac{M_{x x}}{M_{z z}}=\frac{t^{2}\left(1-z^{2}\right)}{2(Q+t z)^{2}} .
$$

So relating this to the longitudinal (density-density) correlation function Eqs. (22) and (23) the transverse correlation function becomes

$$
S_{T}(Q, x)=\hat{S}_{T}(K, k)=\frac{m k_{f}}{4 \pi^{2} \hbar^{2}} I_{T}(Q, x),
$$




$$
I_{T}(Q, x)=\frac{1}{2 Q} \int_{0}^{1}\left[\int_{-1}^{1} \frac{t^{2}\left(1-z^{2}\right)}{\left[x^{2}+(Q+t z)^{2}\right](Q+t z)} d z\right] t^{2} d t .
$$

The exchange energy related to radiation can now be found by utilizing the steps for the electrostatic case where the Coulomb interaction is replaced by the radiating interaction from the $J_{2}$ term of interaction (9). It is

$$
\hat{\psi}_{T}(K, k)=\tilde{\psi}(k) h(K, k), \quad h(K, k)=\frac{-(\omega / c)^{2}}{k^{2}-(\omega / c)^{2}} .
$$

Here $\tilde{\psi}(k)$ is again the Coulomb interaction (9). The frequency $\omega$ is related to the Matsubara frequency via (sign may depend upon convention)

$$
K=i \hbar \omega
$$

With this and new quantities (21) one finds

$$
h(K, k)=\frac{K^{2}}{K^{2}+(c \hbar k)^{2}}=\frac{x^{2}}{x^{2}+Q_{0}^{2}}, \quad Q_{0}=\frac{m c}{\hbar k_{f}},
$$

where with relations (18) one has $Q_{0}^{2}=m c^{2} /\left(2 \mu_{f}\right)$ and $\mu_{f}=50.1 \mathrm{eV} / \mathrm{r}_{\mathrm{s}}^{2}$ by which

$$
Q_{0}=71.5 r_{s}
$$

Like Eq. (17) the radiation excess energy per volume unit can be written as

$$
F_{T e x}=2 \cdot 12 \rho \mu_{f} D I_{\text {Tex }}
$$

where the factor 2 in front is due to equal contributions from both $x$ and $y$ directions. With the modified correlation function and interaction Eq. (24) is modified into

$$
\begin{gathered}
I_{T e x}=\int_{0}^{\infty}\left[\frac{1}{\pi} \int_{-\infty}^{\infty} I_{T}(Q, x) \frac{1}{Q^{2}} \frac{x^{2}}{x^{2}+Q_{0}^{2}} Q d x\right] Q^{2} d Q=\int_{0}^{1}\left[\frac{1}{2} \int_{-1}^{1} J(z, t)\left(1-z^{2}\right) d z\right] t^{4} d t \\
J(z, t)=\int_{0}^{\infty}\left[\frac{1}{\pi} \int_{-\infty}^{\infty} \frac{x^{2} d x}{\left(x^{2}+a^{2}\right)\left(x^{2}+Q_{0}^{2}\right) a}\right] d Q, \quad a=Q+t z .
\end{gathered}
$$

Note that in expression (24) a diverging self-energy part, proportional to density $\rho$, was subtracted. Due to its proportionality to $\rho$ it does not influence the equation of state, i.e. the pressure. This is not so for the present case where such divergence is not present. The result obtained is finite for $Q_{0}>0$. By integration one finds

$$
J(z, t)=\frac{1}{Q_{0}}\left[\ln \left(Q_{0}+|t z|\right)-\ln |t z|\right],
$$




$$
I_{\text {Tex }}=\frac{1}{Q_{0}}\left[\frac{2}{15} \ln Q_{0}+\frac{46}{225}+2 S\right]
$$

where with $\tau=1 / Q_{0}$

$$
\begin{aligned}
S= & \frac{1}{15} \ln (1+\tau)+\frac{1}{8 \tau}[\ln (1+\tau)-\tau]-\frac{1}{12 \tau^{3}}\left[\ln (1+\tau)-\tau+\frac{1}{2} \tau^{2}-\frac{1}{3} \tau^{3}\right] \\
& +\frac{1}{40 \tau^{5}}\left[\ln (1+\tau)-\tau+\frac{1}{2} \tau^{2}-\frac{1}{3} \tau^{3}+\frac{1}{4} \tau^{4}-\frac{1}{5} \tau^{5}\right] .
\end{aligned}
$$

More details of the integrations can be found in the Appendix. For large $Q_{0}$, i.e. $\tau \rightarrow 0$ which means low density, the $S \sim \tau$ and can be neglected. For very small $Q_{0}$, i.e. $\tau \rightarrow \infty$, the two other terms in (44) cancel and one is left with

$$
I_{T e x}=-\frac{1}{4} \ln Q_{0}+\frac{1}{16}+\cdots
$$

One finds that the excess energy due to radiation has opposite sign compared to the electrostatic one. Their ratio is

$$
2 \frac{I_{T e x}}{I_{L e x}}=-8 I_{T e x}
$$

its factor 2 in accordance with Eq. (39). The opposite sign of these excess contributions is as should expected. However, the radiation part exceeds the other for $Q_{0}$ below some value, which from Eq. (38) means small $r_{s}$, i.e. rather high densities. The physical interpretation of this is not clear to us.

\section{CORRELATION ENERGY}

In this section we introduce the correlation energy due to electrostatic and radiation effects.

\section{A. Electrostatic correlation energy}

The Coulomb interaction induces correlations. In the RPA the resulting correlation function becomes [3, 4]

$$
\begin{gathered}
\left.\hat{\Gamma}_{L}(K, k)\right)=\frac{\hat{S}_{L}(K, k)}{1+\hat{A}_{L}(K)} \\
\hat{A}_{L}(K)=g \hat{S}_{L}(K, k) \tilde{\psi}(k)=D \frac{f_{L}(Q, x)}{Q^{2}}
\end{gathered}
$$


with $D$ given by (18). The $f_{L}(Q, x)=I_{L}(Q, x)$ with the latter given by integral (23). The known result is [3, 4, 12]

$$
\begin{aligned}
f_{L}(Q, x)=- & {\left[\frac{Q^{2}-x^{2}-1}{4 Q} \ln \left(\frac{x^{2}+(Q+1)^{2}}{x^{2}+(Q-1)^{2}}\right)\right.} \\
& \left.-1+x \arctan \left(\frac{1+Q}{x}\right)+x \arctan \left(\frac{1-Q}{x}\right)\right] .
\end{aligned}
$$

To obtain more accurate results an effective interaction was introduced in Refs. [3] and [4] $\left(Q=k /\left(2 k_{f}\right)\right.$

$$
\tilde{\psi}_{e}(k)=\tilde{\psi}(k) L(Q)
$$

where $L$ is a function

$$
L(Q)=1-\frac{Q^{2}}{\kappa^{2}}+\cdots
$$

that cuts the interaction for small $r$ in $r$ space, i.e. $L \rightarrow 0$ as $Q \rightarrow \infty$. With effective interaction the $\hat{A}(K)$ in Eq. (47) is replaced by

$$
\hat{A}_{e}(K)=\hat{A}_{L}(K) L(Q)
$$

The results depend somewhat upon the specific form of the function $L$ chosen. In Ref. [4] the parameter $\kappa$ was optimized by thermodynamic self-consistency. One form, which was named Gaussian cut, gave very accurate results.

With the RPA the correlation energy per unit volume is given by [ 3 , 4 ]

$$
F_{\text {Lcorr }}=\frac{1}{2(2 \pi)^{3} 2 \pi} \iint\left[\ln \left(1+\hat{A}_{L}(K)-\hat{A}_{L}(K)\right] d K d \mathbf{k}\right.
$$

With the effective interaction in Eq. (48) and use of thermodynamic self-consistency the precise procedure to obtain $F_{L c o r r}$ is more involved. However, when the parameter $\kappa$ varies little, which was the case, the $\hat{A}_{L}(K)$ may with good accuracy be replaced by quantity (52). After that, the integrand of $(53)$ is divided by $L(Q)$. By evaluations new variables of integration 21 may be used along with relations (18) such that

$$
\frac{d K d \mathbf{k}}{2(2 \pi)^{2} 2 \pi}=12 \rho \mu_{f} \frac{1}{\pi} d x Q^{3} d Q
$$

\section{B. Radiation correlation energy}

Induced correlations and free energy contribution from radiation can be found with precisely the same formalism as for the electrostatic part. With this the correlation function 
and interaction are replaced with the corresponding transverse parts. For the free energy with contributions from both $x$ and $y$ directions one also multiplies with a factor 2 consistent with Eq. (15) for $\left\langle J_{2}\right\rangle$.

Like Eqs. (47) and (48) the transverse correlation function becomes

$$
\begin{gathered}
\left.\hat{\Gamma}_{T}(K, k)\right)=\frac{\hat{S}_{T}(K, k)}{1+\hat{A}_{T}(K)}, \\
\hat{A}_{T}(K)=g \hat{S}_{T}(K, k) \hat{\psi}_{T}(K, k)=D \frac{f_{T}(Q, x)}{Q^{2}} \frac{x^{2}}{x^{2}+Q_{0}^{2}},
\end{gathered}
$$

where $\hat{S}_{T}(K, k)$ is given by Eqs. (33) and (34) and $\hat{\psi}_{T}(K, k)$ is given by Eqs. (35) and (37). To obtain $f_{T}(Q, x)=I_{T}(Q, x)$ integral (34) was performed by analytic evaluation on computer. We found

$$
f_{T}(Q, x)=-\frac{3}{4}+\frac{\left(1+x^{2}-Q^{2}\right)}{2 x}\left[\arctan \left(\frac{1+Q}{x}\right)+\arctan \left(\frac{1-Q}{x}\right)\right]+\frac{\chi(Q, x)}{16 Q x^{2}}
$$

with

$$
\begin{aligned}
\chi(Q, x) & =2\left(1-Q^{2}\right)^{2} \ln \left(\frac{1+Q}{1-Q}\right) \\
& -\left(-2 Q^{2}+Q^{4}-6 Q^{2} x^{2}+\left(1+x^{2}\right)^{2}\right) \ln \left(\frac{(1+Q)^{2}+x^{2}}{(1-Q)^{2}+x^{2}}\right) .
\end{aligned}
$$

Numerical integration of Eq.(57) via Eq.440) produces results for the transverse exchange energy consistent with Eqs. (43) and (44), as is illustrated in Fig. 1. An additional benefit of Eq. (57) is that the wave vector distribution of the transverse exchange energy can be obtained. Then $I_{T}(Q, x)=f_{T}(Q, x)$ is inserted in Eq. (40) and the $x$ integration is performed. By that Eq. (40) turns into the form

$$
I_{T e x}=\int_{0}^{\infty} I_{T}(Q) d Q
$$

where $I_{T}(Q)$ is the result of its $x$ integration. Thus with Eq. (39) the transverse exchange energy per particle and its wave vector distribution $\varepsilon_{T e x}(Q)$ become

$$
f_{T e x}=\frac{F_{T e x}}{\rho}=\int_{0}^{\infty} \varepsilon_{T e x}(Q) d Q, \quad \varepsilon_{T e x}(Q)=24 \mu_{f} D I_{T}(Q) .
$$

In Fig. 3 the $\varepsilon_{T e x}(Q)$ is compared with the corresponding distribution $\varepsilon_{c}(Q)$ for the longitudinal correlation energy from Ref. [4]. 


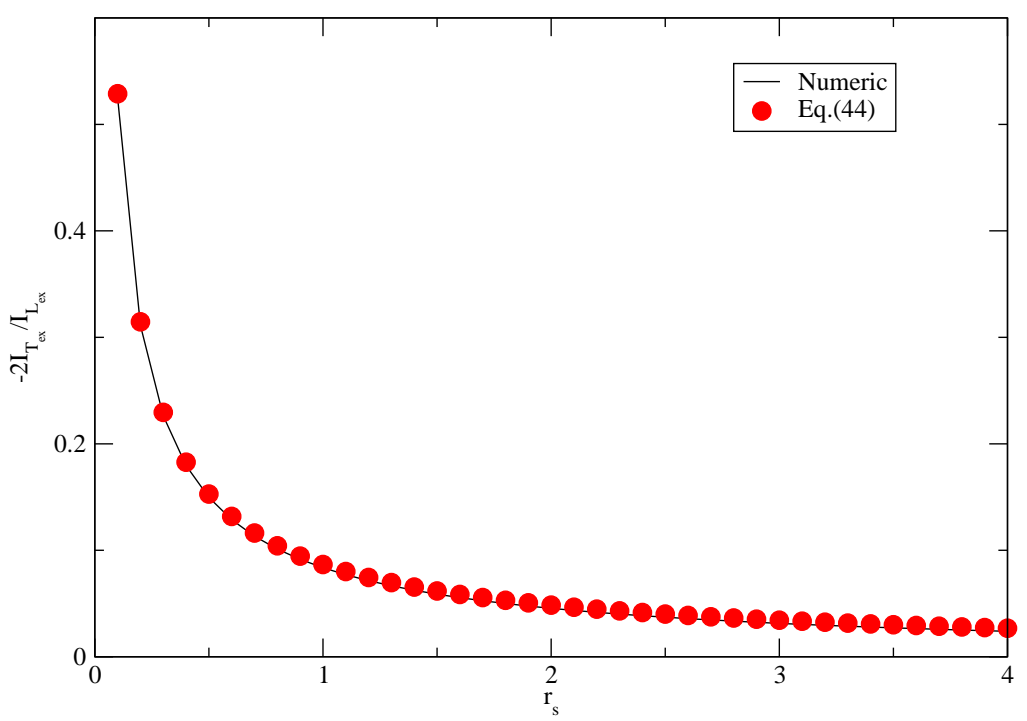

FIG. 1: Relative magnitude of the radiation excess energy compared to the usual electrostatic contribution $-2 I_{\text {Tex }} / I_{\text {Lex }}$ as computed numerically from Eq.(57) and Eq.(40) vs. the analytic expression of Eqs. (43) and (44). Note that $I_{\text {Lex }}$ is given by Eq. 27).

Likewise, from Eq. (53) with the factor 2 included as in Eq. (39), the transverse correlation energy per unit volume becomes

$$
F_{T c o r r}=2 \frac{1}{2(2 \pi)^{3} 2 \pi} \iint\left[\ln \left(1+\hat{A}_{T}(K)-\hat{A}_{T}(K)\right] d K d \mathbf{k}\right.
$$

New variables of integration as given by Eq. (54) are then used. The transverse correlation energy per particle follows from

$$
f_{T c}=\frac{F_{T c o r r}}{\rho} .
$$

From Fig. 2 it is seen that $f_{T c}$ is very small.

The use of effective interaction with a cut function $L(Q)$ as sketched below Eq. (53) may also be used for $F_{\text {Tcorr }}$. However, since the latter is expected to be a very small correction to this already small quantity, it will be of little significance anyway. So we have not investigated this further. 


\section{DIELECTRIC CONSTANT}

The uniform electron gas can be thought as the free conduction electrons of metals, and and so it can be regarded as a dielectric fluid. In our derivations the electron gas has already been treated as such a fluid. Now the dielectric properties are tightly connected to the pair correlation function that can be seen as a resulting interaction in the dielectric medium.

The direct interaction between polarizations $\mathbf{s}_{1}$ and $\mathbf{s}_{2}$ in vacuum is given by expressions (9) and (10). In a dielectric medium the corresponding interaction should be

$$
\begin{gathered}
\hat{\phi}_{\varepsilon}(12, \omega)=\hat{\psi}_{\varepsilon L}(k, \omega) J_{1}+\hat{\psi}_{\varepsilon T}(k, \omega) J_{2} \\
\hat{\psi}_{\varepsilon L}(k, \omega)=\frac{\tilde{\psi}(k)}{\varepsilon_{L}}, \quad \hat{\psi}_{\varepsilon T}(k, \omega)=\frac{\tilde{\psi}(k)}{\varepsilon_{T}} \frac{-\Omega^{2}}{k^{2}-\Omega^{2}}, \quad \Omega^{2}=\varepsilon_{T}\left(\frac{\omega}{c}\right)^{2},
\end{gathered}
$$

where $\varepsilon_{L}$ and $\varepsilon_{T}$ are longitudinal and transverse dielectric constants. They are expected to be equal in the continuum limit of small wave vectors, $k \rightarrow 0$, and nonzero frequencies. For the electrostatic case $\omega=0$ the transverse part clearly does not contribute.

The interactions in a dielectric medium is given by the direct interaction (9) plus induced contributions via the correlated particles of the dielectric medium. This involves the same convolutions used to obtain the resulting correlation functions (47) and (55). Thus $(K=$ $i \hbar \omega)$

$$
\hat{\psi}_{\varepsilon L}(k, \omega)=\frac{\tilde{\psi}(k)}{1+\hat{A}_{L}(K)}, \quad \hat{\psi}_{\varepsilon T}(k, \omega)=\frac{\hat{\psi}_{T}(K, k)}{1+\hat{A}_{T}(K)} .
$$

Comparing with Eq. (64) one finds

$$
\varepsilon_{L}=1+\hat{A}_{L}(K)=1+g \hat{S}_{L}(K, k) \tilde{\psi}(k), \quad \varepsilon_{T}=1+g \hat{S}_{T}(K, k) \tilde{\psi}(k)
$$

The result for $\varepsilon_{T}$ follows by noting that it implies $1+\hat{A}_{T}(K)=1+\left(\varepsilon_{T}-1\right) h(K, k)$ with $h(K, k)$ given by (35). One may also use the cut interaction (50) which implies multiplying the $\tilde{\psi}(k)$ in 67 with $L(Q)$. Anyway, the concept of dielectric constant is mainly useful only in the long wavelength continuum limit, i.e. $k \rightarrow 0$, where the particle structure on the microscopic scale is not present. In this limit where $x \rightarrow \infty$ and $Q \rightarrow 0$ integral (23) becomes

$$
I_{L}(Q, x)=\frac{2}{3 x^{2}}
$$


When inserted in Eq. (22) and then inserted in (67) this results in

$$
\varepsilon_{L}=1-\left(\frac{\omega_{p}}{\omega}\right)^{2}, \quad \omega_{p}^{2}=\frac{\rho e^{2}}{m \varepsilon_{0}},
$$

where Eqs. (10), (18), and (21) are used. The $\omega_{p}$ is the plasma frequency. This result is the well known expression for the dielectric constant due to the free electrons in metals.

The corresponding situation with integral (34) for the transverse case is less straightforward. But one can write

$$
\frac{t^{2}\left(1-z^{2}\right)}{Q+t z}=\frac{t^{2}}{Q+t z}+Q-t z-\frac{Q^{2}}{Q+t z}
$$

By integration with respect to $z$ only the first and second terms contribute as the last one is of higher order in $Q$. The $z$-integration of these two terms gives $t \ln [(t+Q) /(t-Q))]+2 Q \rightarrow 4 Q$, $Q \rightarrow 0$. When used in integral (34) the results is

$$
I_{T}(Q, x)=\frac{2}{3 x^{2}}
$$

Accordingly in the limit $k \rightarrow 0$ ( $\omega$ finite)

$$
\varepsilon_{T}=\varepsilon_{L}
$$

\section{RESULTS}

The transverse correlation energy per particle 62 can be evaluated by numerical integration of Eq. (61), using the transformation (54), and inserting Eqs.(56) and (57). The double integration over $\mathrm{Q}$ and $\mathrm{x}$ is carried out using 10000 grid points for each variable and grids $\Delta Q=\Delta x=0.01$. The numerical integration via Eq. (57) has been checked against the analytical results for the evaluation of the radiation excess energy in Figure 1.

In Figure 2 both the transverse excess energy due to radiation and the transverse correlation energy is presented and compared with the correlation energy determined from the Perdew-Wang fit [13] and the Gaussian cut approximation from Ref. [4]. All these quantities are scaled with the longitudinal excess energy (17). One can readily see that the transverse contribution is mostly a small fraction of the longitudinal one. Relative to the latter it decays close to an inverse power of $r_{s}$ for decreasing density $\left(\rho \sim 1 / r_{s}^{3}\right)$, but growing for very high densities according to Eq. (45). As seen from the figure, the transverse correlation energy is very small compared to the excess part. 


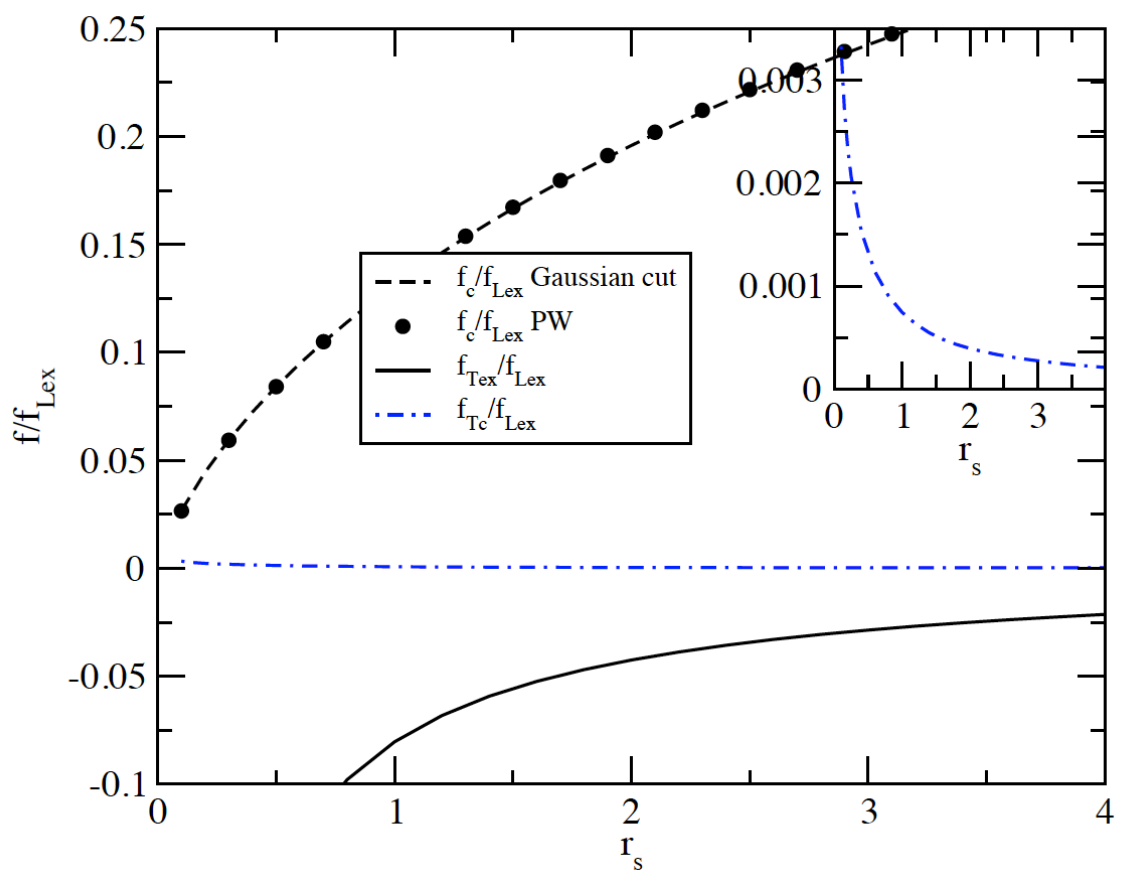

FIG. 2: Transverse excess and correlation energies per particle $f_{T e x}=F_{T e x} / \rho$ and $f_{T c}$ given by Eqs. (39) and (62) respectively. This is related to the Perdew-Wang fit [13] to the longitudinal electrostatic correlation energy per particle $f_{c}=F_{L c o r r} / \rho$ and the Gaussian cut approximation to it from Ref. 44. All curves for $f=f_{c}, f_{T e x}$, and $f_{T c}$ respectively are scaled with the longitudinal excess energy (16) and (17) with $f_{\text {Lex }}=F_{\text {Lex }} / \rho$. The Gaussian cut $F_{L c o r r}$ was found as sketched below Eq. (53).

The behavior of the transverse excess energy contribution from radiation is illustrated by the wave vector analysis of it in Fig. 3. There the wave vector distribution $\varepsilon_{\text {Tex }}(Q)$ of Eq. (60) is plotted. It is compared with the corresponding analysis of the longitudinal electrostatic correlation energy from the Perdew-Wang fit [13] and the Gaussian cut approximation from Ref. [4]. One can see that for $r_{s}$ characteristic of aluminum (one of the smallest, $\sim 2$ ) the correction due to radiation is hardly noticeable. However, for much higher densities $\left(r_{s}=0.5\right)$ the correction is considerable where also values for $Q=k /\left(2 k_{f}\right)>1$ give a significant contribution. 


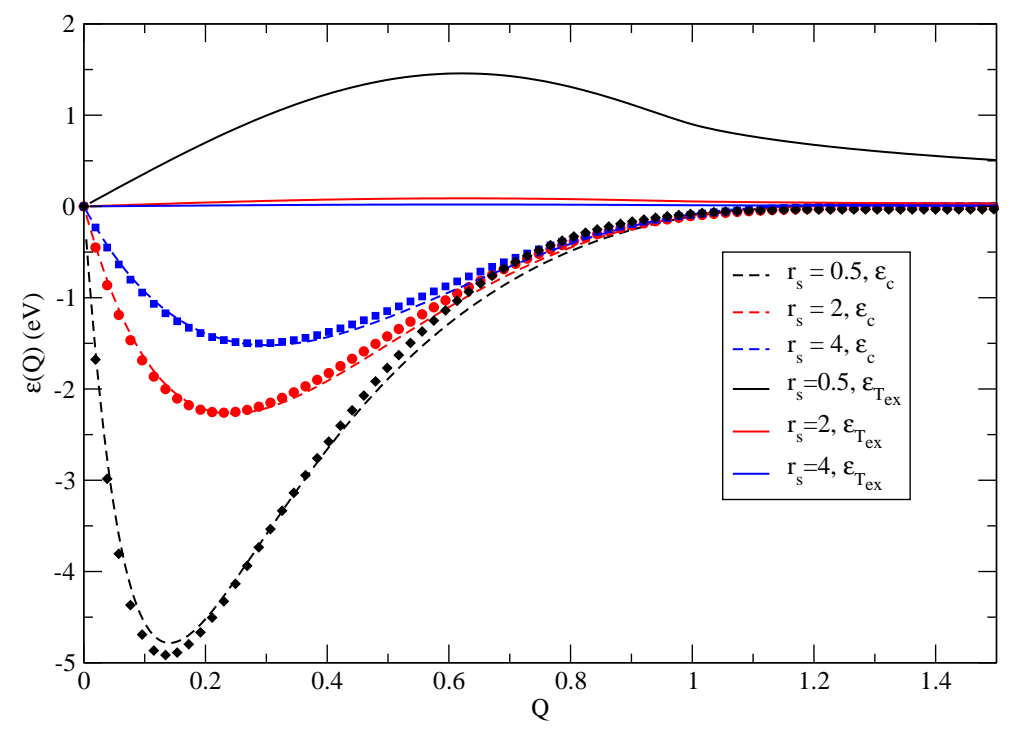

FIG. 3: Wave vector analysis of the electron transverse excess energy due to radiation where its distribution $\varepsilon(Q)=\varepsilon_{\text {Tex }}(Q)$ from Eq. (60) is plotted (fully drawn curves). This is compared with a corresponding plot of the longitudinal correlation energy $\varepsilon(Q)=\varepsilon_{c}(Q)$ computed from the Perdew-Wang fit [13] (dots) and the Gaussian cut approximation from Ref. [4] (dashed curves).

\section{Summary}

In this work we have obtained radiation corrections to the (free) energy of the quantized uniform electron gas at $T=0$. It is positive and adds to the negative electrostatic Coulomb energy to reduce it. In comparison its relative magnitude is small, except for rather large densities. It decreases rapidly with decreasing densities. The greater part of it is the transverse excess energy. This energy comes from the coupling of the radiating electromagnetic field to the current correlations of the free unperturbed fermion gas of electrons. This coupling also induces transverse contributions to the correlation function that give the transverse correlation free energy. But this free energy contribution turns out to be very small. Further the induced longitudinal electrostatic and transverse correlation functions defines non-local longitudinal and transverse dielectric constants of the uniform electron gas. As should be expected they are equal and in accordance with the well-known plasma relation for oscillations in the electron gas in the long wavelength limit $(k \rightarrow 0)$. 


\section{Appendix}

Here details of the evaluation of integral 40 is given. Expression (42) for $J(z, t)$ makes the integrand symmetric around $z=0$ by which $z$ can be restricted to lie between 0 and 1 . This is compensated by multiplication with 2 and the integral becomes

$$
I_{T e x}=\int_{0}^{1} \int_{0}^{1} \frac{1}{Q_{0}}\left[\ln \left(Q_{0}+t z\right)-\ln (t z)\right]\left(1-z^{2}\right) d z t^{4} d t .
$$

The last ln integral is easily obtained by partial integrations $\left(\int_{0}^{1} u^{n} \ln u d u=-1 /(n+1)^{2}\right)$

$$
-\int_{0}^{1} \int_{0}^{1}(\ln t+\ln z)\left(1-z^{2}\right) d z t^{4} d t=\frac{46}{225} .
$$

The first ln integral will be more cumbersome by partial integrations. However, we choose to expand the ln term in the quantity $t z \tau$ where

$$
\begin{gathered}
\tau=\frac{1}{Q_{0}} . \\
\ln \left(Q_{0}+t z\right)=\ln Q_{0}+\sum_{n=1}^{\infty} \frac{(t z \tau)^{n}}{n}(-1)^{n-1} .
\end{gathered}
$$

By integration of the resulting power series the two $z$ integrations result into the factor

$$
\frac{1}{n+1}-\frac{1}{n+3}=\frac{2}{(n+1)(n+3)}
$$

while the $t$ integration gives the factor $1 /(n+5)$. Finally $\int_{0}^{1} \int_{0}^{1}\left(1-z^{2}\right) d z t^{4} d t=2 / 15$. Altogether we find

$$
\begin{aligned}
I_{\text {Tex }} & =\frac{1}{Q_{0}}\left(\frac{2}{15} \ln Q_{0}+\frac{46}{225}\right)+\frac{2}{Q_{0}} S, \\
S & =\sum_{n=1}^{\infty} \frac{(-1)^{n-1} \tau^{n}}{n(n+1)(n+3)(n+5)} .
\end{aligned}
$$

The series for $S$ may be summed, and expansion in partial fraction gives

$$
\frac{1}{n(n+1)(n+3)(n+5)}=\frac{1}{15 n}-\frac{1}{8(n+1)}+\frac{1}{12(n+3)}-\frac{1}{40(n+5)} .
$$

With this four logarithmic series are obtained as given by expression (44) for $S$. 


\section{Acknowledgment}

EL acknowledges the support from the Agencia Estatal de Investigacin and Fondo Europeo de Desarrollo Regional (FEDER) under grant No. FIS2017-89361-C3-2-P. JSH acknowledges financial support from the Research Council of Norway, Project 250346.

[1] P. Fulde, Electron Correlation in Molecules and Solids, 2nd Ed. (Springer, Berlin, 1993).

[2] J.S. Høye, Phys. Rev. E 81, 061114 (2010).

[3] J.S. Høye and E. Lomba, J. Phys.; Condens. Matter 28, 41400 (2016).

[4] E. Lomba and J.S. Høye, J. Phys.; Condens. Matter 29, 435 (2017).

[5] M.S. Wertheim, J. Chem. Phys. 55, 4291 (1971).

[6] J.S. Høye, J.L. Lebowitz, and G. Stell, J. Chem. Phys. 61, 3253 (1974).

[7] J.S. Høye, G. Stell, J. Chem. Phys. 77, 5173 (1982).

[8] L. Blum and A. J. Torruella, J. Chem. Phys. 56, 303 (1972)

[9] M.H. Waage and J.S. Høye, AIP Advances 3, 022118 (2013).

[10] H.B.G. Casimir and D. Polder, Phys. Rev. 73, 360 (1948).

[11] I. Brevik and J.S. Høye, Physica A 153 (1988).

[12] M. Lein, E.K.U. Gross, and J.P. Perdew, Phys. Rev. B 61, 13431 (2000).

[13] J. P. Perdew, Y. Wang, Phys. Rev. B, 45, 13244 (1992); ibidem, 46, 12947 (1992) 